Cleveland, United States; ${ }^{6}$ University Medical Center, Groningen, Netherlands; ${ }^{7}$ Johns Hopkins University, Baltimore; ${ }^{8}$ Hospital for Special Surgery, New York; ${ }^{9}$ Duke University Medical Center, Durham; ${ }^{10}$ Massachusetts General Hospital; ${ }^{11}$ Boston University, Boston, United States

Background: Evidence supporting the classification of anti-neutrophil cytoplasmic antibody (ANCA)-associated vasculitides (AAV) based on ANCA type is accumulating. ${ }^{1}$

Objectives: To evaluate serum cytokine profiles in patients with AAV classified by ANCA specificity (proteinase 3 (PR3)-ANCA versus myeloperoxidase (MPO)ANCA) or by clinical diagnosis (granulomatosis with polyangitis (GPA) versus microscopic polyangiitis (MPA)) and clinical phenotypes.

Methods: An antibody array testing 30 soluble mediators, already shown to be implied in the pathogenesis of AAV, was performed in each patient with active AAV at inclusion in the Rituximab in ANCA-Associated Vasculitis (RAVE) trial, as previously described. ${ }^{2,3}$ By means of Wilcoxon signed rank test for univariate analyses, we analyzed the association of levels of these cytokines with ANCA specificity, clinical diagnosis, and distinct clinicopathologic phenotype categories derived from the BVAS/WG items recorded at the time of enrollment (capillaritis, granulomatous manifestations, renal involvement, and alveolar hemorrhage; new diagnosis and relapsing disease), as described. ${ }^{4}$

Results: All cytokines tested (see Figure 1 and legend for the complete list), except for RANTES, ACE, bFGF and VCAM-1, were significantly increased in the RAVE cohort when compared to healthy controls $(p<0.05)$. Median Birmingham vasculitis activity score and steroid use at screening did not significantly differ between PR3-AAV and MPO-AAV, and between GPA and MPA. Within both ANCA specificities, levels of 9 mediators were significantly higher in PR3-AAV (IL-6, NGFb, GM-CSF, IL-15, IL-18, IL-18Bb, sIL-2Ra, IL-8, TARC; $p<0.05$ ), compared to 5 different cytokines that were higher in MPO-AAV (sIL6R, NGAL, SICAM-1, VCAM-1, sTNFR II; $p<0.05$ ). In contrast, only 4 cytokines (GM-CSF, IL-15, IL-18, sIL-2Ra) were higher in GPA than MPA, and 1 (NGAL) was higher in MPA than GPA $(p<0.05)$. The association of the majority of cytokines was stronger with ANCA specificity than with the clinical diagnosis (Figure 1). Similarly, the defined clinical phenotypes were also not separated by cytokine signatures as clearly as the ANCA specificity was (data not shown). Restricting the analysis to newly-diagnosed patients $(n=90)$ showed the most significant cytokine profile differences associated with PR3-AAV (Figure 1).

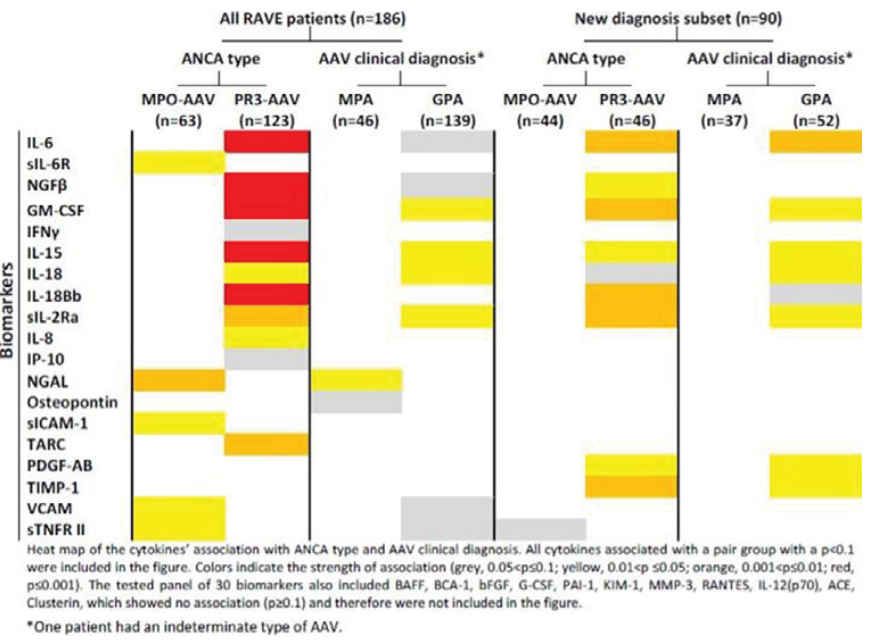

Conclusions: Cytokine profiles separate patients more clearly by ANCA specificity than by clinical diagnosis, suggesting important differences in underlying pathophysiology and validating stratification of patients by ANCA specificity for treatment trials.

References:

[1] Cornec D et al. Nat Rev Rheumatol. 2016 Oct;12(10):570-9.

[2] Monach PA et al. Ann Rheum Dis. 2013 Aug;72(8):1342-50.

[3] Stone J et al. N Engl J Med. $2010 \mathrm{Jul}$ 15;363(3):221-32.

[4] Fussner LA et al. Arthritis Rheumatol. 2016 Jul;68(7):1700-10.

Disclosure of Interest: None declared

DOI: 10.1136/annrheumdis-2017-eular.5672

\section{FRI0315 CLINICAL CHARACTERISTICS OF VASCULAR INVOLVEMENT IN BEHCET'S DISEASE}

A. Ates, M. Torgutalp, E. Okatan, E. Uslu, B. Dinçer, E. Yayla, M. Turgay, G. Kınıklı. Rheumatology, Ankara University School of Medicine, Ankara, Turkey

Background: Behçet's disease (BD) is a multisystemic inflammatory disorder classified as vasculitis, which can affect all types of vessels. The prevalence of vascular involvement has been reported at rates ranging from 15 to $50 \%$.

Objectives: In this study, we aimed to determine the characteristics of vascular involvement in patients with $\mathrm{BD}$.
Methods: Six hundred and ninety-two patients with BD, who applied to the multidisciplinary BD policlinic between 2006-2016, were retrospectively analyzed. The diagnosis of vascular involvement was made on clinical signs, by Doppler ultrasonography and/or angiography using computed tomographic or magnetic resonance techniques where appropriate.

Results: One hundred and seventy-six patients (\%25.4) had vascular involvement. The mean age of patients with vascular involvement $(n=176)$ and non-vascular involvement $(n=516)$ was similar to $42.5 \pm 12.3$ and $42.2 \pm 12.2$ years, respectively, while male sex frequency was significantly higher in patients with vascular involvement $(72 \%$ to $\% 42, \mathrm{p}<0.001)$. Uveitis $(45 \%)$ and erythema nodosum (EN) $(47.7 \%)$ were significantly higher in patients with vascular involvement than those without vascular involvement (for Uveitis OR: 1.7, $\mathrm{p}=0.003 /$ for $E N$ : OR: $2.5, p<0.001$ ) (Table 1). Deep vein thrombosis was detected in $142(80.7 \%)$ of the patients with vascular involvement and this was the most common type of vascular involvement (Table 2). Arterial involvement was detected in 52 (29.5\%) of 176 patients with vascular involvement. Deep venous thrombosis alone was present in $125(71 \%)$ patients, arterial involvement alone in $12(6.8 \%)$ patients, arterial and venous involvement together detected in $40(22.7 \%)$ patients. Arterial involvement was most commonly seen as pulmonary artery involvement in 45 $(25.6 \%)$ patients [pulmonary artery thrombosis $35(20 \%)$, aneurysm $10(5.7 \%)$ and thrombosis + aneurysm $6(3.4 \%)$ patients]. Twenty (11.3\%) patients had other arterial involvement except pulmonary, 8 of them had thrombosis and 12 had aneurysm.

Table 1. Clinical features in patients with and without vascular involvement

\begin{tabular}{lccccc}
\hline & $\begin{array}{c}\text { With vascular } \\
\text { involvement }\end{array}$ & $\begin{array}{c}\text { Without vascular } \\
\text { involvement }\end{array}$ & OR & $95 \% \mathrm{Cl}$ & $\mathrm{P}$ value \\
\hline $\mathrm{n}(\%)$ & $176(25.4)$ & $516(74.6)$ & & & \\
Age (year) & $42.5 \pm 12.3$ & $42,2 \pm 12.2$ & & & 0.762 \\
Male n (\%) & $127(72.2)$ & $217(42.1)$ & 3.57 & $2.46-5.19$ & $<0.001$ \\
Genital ulceration n (\%) & $123(69.9)$ & $392(76.1)$ & 0.728 & $0.498-1.065$ & 0.101 \\
$\begin{array}{l}\text { Uveitis n (\%) } \\
\text { Papulopustular erythema or }\end{array}$ & $79(44.9)$ & $166(32.4)$ & 1.698 & $1.196-2.409$ & $\mathbf{0 . 0 0 3}$ \\
$\quad$ acneiform rash n (\%) & $101(51.4)$ & $186(57.4)$ & 1.274 & $0.886-1.832$ & 0.190 \\
EN n (\%) & $84(47.7)$ & $98(27.1)$ & 2.460 & $1.689-3.582$ & $<0.001$ \\
\hline
\end{tabular}

Table 2. Venous involvement (\%)

Deep vein thrombosis

Budd-Chiari syndrome

lliac vein thrombosis

Safenous vein thrombosis

Femoral vein thrombosis

Popliteal vein thrombosis

Crural vein thrombosis

Cerebral sinus thrombosis

$142(80.7)$
$7(4.0)$
$26(14.7)$
$18(10.2)$
$23(13.1)$
$36(20.5)$
$43(24.4)$
$12(6.8)$
$20(12,2)$

Conclusions: Vascular involvement in Behçet's disease is commonly seen in males. Deep vein thrombosis is the most common form of vascular involvement. Arterial involvement is important because of its higher risk of mortality (especially pulmonary artery aneurysm). The incidence of EN and uveitis is higher in patients with vascular involvement.

Disclosure of Interest: None declared

DOI: 10.1136/annrheumdis-2017-eular.4225

\section{FRI0316 FACTORS ASSOCIATED WITH SURGICAL OUTCOMES OF SEVERE AORTIC REGURGITATION IN PATIENTS WITH BEHCET'S DISEASE}

B. Ghang ${ }^{1}$, D.-H. Lim ${ }^{2}$, W.J. Seo ${ }^{3}$, Y.-G. Kim ${ }^{1}$, B. Yoo ${ }^{1} .{ }^{1}$ Rheumatology, Asan medical center, Seoul; ${ }^{2}$ Rheumatology, Ulsan University Hospital, Ulsan

${ }^{3}$ Rheumatology, Seoul Veterans Hospital, Seoul, Korea, Republic Of

Background: In rare cases, Behcet's disease (BD) can cause severe aortic regurgitation $(\mathrm{AR})$ or aortic root destruction that might have lethal outcomes. Conventionally, simple aortic valve replacement (AVR) is performed for the management of severe AR in BD patients; however, the reoperation rate is as high as 78 to $100 \%$, while mortality rate range from 20 to $47 \%$. Recently, several series of case reports showed that compared to AVR, aortic root replacement (ARR) improved the surgical outcomes of AR in BD patients.

Objectives: To identify the factors associated with the long-term surgical outcomes of $A R$ in $B D$ patients.

Methods: We identified 23 patients who had been surgically treated for AR caused by aortic root involvement of BD from January 1996 through December 2013. We evaluated the occurrence of post-surgical adverse events, which were defined as follows: death, aortic valve/graft problem, infective endocarditis, cerebral infarction, and/or re-operation of aortic valve or root. Types of surgery were classified as simple aortic valve replacement (AVR), bioprosthesis aortic root replacement (bARR), and mechanical valved composite graft aortic root replacement (cARR). Clinical parameters including baseline characteristics, C-reactive protein (CRP), erythrocyte sedimentation rate, and medications were extracted from electronic medical records.

Results: Appropriate aortic valve or root surgery cases were 35 in total, with a mean follow up duration of $11 \pm 5$ years in 23 patients. Out of the 11 cases 